\title{
Sum of Sine Modeling Approach as a New Processing Technique For a Biometric System Based on ECG Signal
}

\author{
Abbas Abou Ahmad \\ Department of Biomedical Engineering \\ Islamic University of Lebanon \\ Beirut, Lebanon \\ a.abouahmad@gmail.com
}

\author{
Mohammad Ayache \\ Department of Biomedical Engineering \\ Islamic University of Lebanon \\ Beirut, Lebanon \\ mohammad.ayache@iul.edu.lb
}

\author{
Alaa Daher \\ Faculty of Engineering \\ Beirut Arab University \\ Debbieh, Lebanon \\ a.daher@bau.edu.lb
}

Received: September 30, 2020. Revised: November 30, 2020. Accepted: December 4, 2020. Published: December 9, 2020.

\begin{abstract}
Biometrics is considered in current research as one of the best methods for authenticating human beings. In our paper, the heartbeat biometric, also called Electrocardiographic (ECG), is working on. This biometric is chosen because human ECGs cannot be falsely created and replicated. This study aims to find the best features from this biometric that can identify a person, given the extractions and classification algorithms for the heartbeat biometric signal. Depending on a literature study we work to propose a new and more efficient technique based on a new method for ECG features extraction and these features will be the inputs for pattern recognition classifier. This methodology will be tested on real experimental ECG data that is collected.

The Data collected from 10 subjects by a commercial ECG device taking the data from lead 1. The pre-processing steps start with the Empirical Mode Decomposition (EMD) before digital filters which are: low pass, high pass, and derivative pass filters. Features extraction steps are peak detection, segmentation, and wave modeling for each segment. The classification used the Multi-Layer Perceptron and compared it to classification using Radial Basis Function were the results of MLP were much better for these applications since the accuracy of the final results of MLP is $99 \%$ and that related to the RBF is $95 \%$.
\end{abstract}

Keywords-Biometrics, Electrocardiogram, Waves Modeling, Neural Network.

\section{INTRODUCTION}

Authentication in human technologies is very important today since life engages technology in multiple ways. There is a high demand for secure and reliable authentication. However, some traditional methods for authentication such as passwords and tokens are now outdated since there is a high possibility to be stolen or to lose.

ECG signal is a universal characteristic. This signal has been used for several decades as an effective tool for diagnostic in medical applications. Recently, there are suggestions for using this ECG signal as a biometric tool. Its validity is well supported by the fact that both the physiological and geometrical differences of the heart under different subjects reveal certain uniqueness in the signal characteristics [1].

Due to the differences in morphology among individuals, the ECG signal for each subject contains a unique pattern. The Electrocardiogram (ECG) is the electrical activity recording of the human heart over a while. The waveform shape shows the current state of the heart and it gives helpful information regarding the heart rhythm and function. One cardiac cycle in an ECG signal consists of the P-QRS-T Characteristic waves [2].
ECG has the following characteristics: Universality, Uniqueness, Stability, Collectability, Performance, Acceptability, and Circumvention. These characteristics are needed for a biometric to be applicable for access control, thus our target of using the ECG as a biometric tool to identify individuals can be applied.

So for this purpose, we use a new method of application starting from the data pre-processing that uses mainly the EMD and then the digital filters and not ending with the features extraction that applies the peak detection and use these peaks to segment the signal and finally make the model of each part of the wave using multi-sine mode. For classifying the signals and so the individuals, we apply two types of methods which are the Multi-layer Perceptron (MLP) and Radial Basis Function (RBF).

The organization of this paper is as follows:

- Literature of Review

- Materials and methods showing the data acquisition and pre-processing then the method used for feature extraction and finally the classification and identification of the individual.

- Results of classification and discussion.

- Conclusion of all the above work done.

\section{LITERATURE OF REVIEW}

ECG-based recognition approaches are numerous and very different. ECG attributes (features) are intended to classify the specific subject exploiting inter-subject variability. No agreement exists on the most appropriate technique or on the type/number of features to consider.

Below are some of the researches that use ECG to identify individuals from 1998 till 2019:

Biel et al. [3] used temporal features, Modeling Methodology uses Principal Component Analysis (PCA).

Shen et al. [4] used 7 fiducial ECG-features. They then classify the features with a combination of template matching and decision based neural network (DBNN).

Israel et al. [5] used Amplitude features. The classification was performed using standard Linear Discriminant Analysis (LDA).

Gahi et al. [6] extracts 24 temporal and amplitude features from an ECG signal and after processing, reduces the set of features to the nine most relevant features. The selected features are compared to the stored templates to generate a matching score and classify the smallest distance. 
Safie et al. [7] derived fiducial features and then classification handled by Euclidean distance and KNN.

Iqbal et al. [8] extract features using the Cardioid graphbased model. In this model, features were extracted solely from the graph derived from the QRS complexes. Subjects were classified by Multilayer Perceptron (MLP).

Janani et al. [9] handle BOTH fiducial and non-fiducial ECG features. Bayesian networks beside KNN classifiers led to a better recognition performance when one type of classifier was used.

Abd ElRaheem et al. [10] uses the Equal Distance descriptor for identification, and selected Fourier Descriptor coefficients of the main loop of the Vector Cardiogram VCG are used as biometric data Feed Forward Neural Networks are used as classifiers.

Plataniotis et al. [11] have proposed an approach that uses AutoCorrelation Analysis (AC) coupled with Discrete Cosine Transform (DCT) to classify the individuals using the nonfiducial features.

Chiu et al. [12] extracted the features by applying the discrete wavelet transform. The Euclidean distance was used as a measure for the verification mechanism. With Haar wavelet, wavelet coefficients decomposed from the signal were used as the biometric "identity card" of the subject.

Chan et al. [13] classify the individuals using three different distances: percent residual difference (PRD), correlation coefficient (CCORR), and WDIST were all measures the difference between coefficients of two ECGs.

Zeng et al. [14] use a statistical-based algorithm called the reduced binary pattern (RBP). This algorithm converts signals into concise binary patterns and performs statistical counting and ranking for identification.

Dar et al. [15] proposed the extraction of features from Haar transform and heart-rate-variable RR intervals. The method chosen for the detection of R-peaks was thresholding of local maxima. The decision method was based on $\mathrm{k}$ Nearest Neighbours (kNN).

Pinto et al. [16] extract Discrete Cosine Transform (DCT) and Haar transform features and fed to decision methods based on Support Vector Machines (SVM), k-Nearest Neighbours (kNN), Multilayer Perceptrons (MLP), and Gaussian Mixture Models.

Kim et al. [17] used Haar-wavelet transform (HWT) to extract ECG biometric features. The classifier of the neural network with weighted fuzzy membership functions (NEWFM) is used.

00 Huang et al. [18] use non-fiducial point extraction and high order autocorrelation. Using the improved AC algorithm combined with the method of DCT to realize feature extraction. Then using discrete cosine transform to reduce the dimension of the autocorrelation data. Finally, using the KNN classifier to realize identification. Table 1 resumes the different techniques used for ECG identification and the results obtained.
Table 1: Comparison of different techniques in ECG based biometrics

\begin{tabular}{|c|c|c|c|c|c|}
\hline Author & Year & Features & Methodology & Subjects & Accuracy \\
\hline $\begin{array}{l}\text { Biel et } \\
\text { al. }{ }^{[9]}\end{array}$ & $\begin{array}{l}1999- \\
2001\end{array}$ & Fiducial & PCA & 20 & $100 \%$ \\
\hline $\begin{array}{l}\text { Shen et } \\
\text { al. }{ }^{[10]}\end{array}$ & 2002 & Fiducial & Template Matching + DBNN & 20 & $100 \%$ \\
\hline $\begin{array}{l}\text { Israel et } \\
\text { al. }{ }^{[11]}\end{array}$ & 2005 & Fiducial & LDA & 29 & $98 \%$ \\
\hline $\begin{array}{l}\text { Gahi et } \\
\text { al. }{ }^{[12]}\end{array}$ & 2008 & Fiducial & Template Matching & 16 & $100 \%$ \\
\hline $\begin{array}{l}\text { Safie et } \\
\text { al. } .^{[13]}\end{array}$ & 2011 & Fiducial & Euclidean distance $+\mathrm{KNN}$ & 112 & $91 \%$ \\
\hline $\begin{array}{l}\text { Iqbal et } \\
\text { al. }{ }^{[14]}\end{array}$ & 2014 & Fiducial & MLP & 30 & $96.4 \%$ \\
\hline $\begin{array}{l}\text { Janani, } \\
\text { David et } \\
\text { al. }{ }^{[15]}\end{array}$ & 2009 & Both & $\mathrm{KNN}+$ Bayesian & 17 & $88 \%$ \\
\hline $\begin{array}{l}\text { Abd- } \\
\text { elraheem } \\
\text { et al. }{ }^{[16]}\end{array}$ & 2012 & Both & Fourier Descriptor Coef. + VCG & 22 & $99.45 \%$ \\
\hline $\begin{array}{l}\text { Platanioti } \\
\text { set al. }{ }^{[5]}\end{array}$ & 2006 & Non Fiducial & Autocorrelation + DCT & 14 & $100 \%$ \\
\hline $\begin{array}{l}\text { Chiu et } \\
\text { al. }{ }^{[17]}\end{array}$ & 2008 & Non Fiducial & Wavelet Distance + LDA & 35 & $100 \%$ \\
\hline $\begin{array}{l}\text { Chan et } \\
\text { al. }{ }^{[18]}\end{array}$ & 2008 & Non Fiducial & Wavelet DM & 50 & $95 \%$ \\
\hline $\begin{array}{l}\text { Zeng et al. } \\
\text { [19] }\end{array}$ & 2012 & Non Fiducial & Reduced binary pattern & 48 & $95.79 \%$ \\
\hline $\begin{array}{l}\text { Dar et } \\
\text { al. }{ }^{[20]}\end{array}$ & 2015 & Non Fiducial & Haar Transform + KNN & 47 & $93.1 \%$ \\
\hline $\begin{array}{l}\text { Pinto et } \\
\text { al. }[21]\end{array}$ & 2017 & Non Fiducial & $\mathrm{DCT}+\mathrm{SVM}$ & 6 & $94.9 \%$ \\
\hline $\begin{array}{l}\text { Kim et } \\
\text { al. }{ }^{[22]}\end{array}$ & 2018 & Non Fiducial & Haar Transform + FNN & 73 & $98.3 \%$ \\
\hline $\begin{array}{l}\text { Huang et } \\
\text { al. }{ }^{[32]}\end{array}$ & 2019 & Non Fiducial & $\mathrm{DCT}+\mathrm{KNN}$ & 294 & $99.7 \%$ \\
\hline
\end{tabular}

\section{MATERIALS AND METHODS}

\section{A. Data Acquisition}

The ECG data used in this research is obtained from real collected data. The number of data is 10 subjects recorded over 210 seconds long. The subjects are six men aged 20 to 30 years and four women with ages 20 to 25 years. The ECGs were taken by a commercial ECG device $(500 \mathrm{~Hz}$ as a sampling rate and $500 \mathrm{~dB}$ for gain). The recordings for each subject are obtained from ECG lead 1. In this step, the signal is taken and stored in the database, which will be used by the system for matching.

\section{B. Data Pre-Processing}

ECG data collected usually contain many types of noise. Due to the presence of this noise, feature extraction and classification may occur with less accuracy. For the ideal data structure, we must process the raw ECG. First, we have to identify noisy sources [19]. In our research, digital filters (low pass filter, high pass filter, and derivative base filter), as shown in figure 1 below, are used to remove the major three noises of baseline drift, power line interference, and EMG noise.

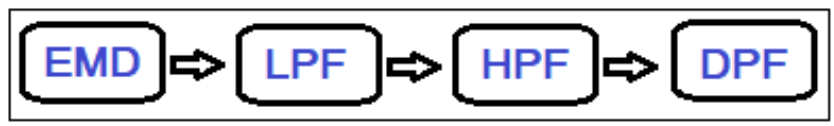

Figure 1: Data Pre-Processing Block Diagram

EMD is a technique of decomposition which allows representing a signal through a sum of functions derived from the latter, called Intrinsic Mode Function (IMF). The decomposition of the signal by EMD is the fundamental step to obtain the Hilbert spectral analysis. 
The individual IMFs are obtained by an iterative operation which is a sifting operation. The main steps to have the IMF decomposition are:

a. Local extremes identification. In particular, the local values of maximum and minimum of the signal should be separately evaluated.

b. The upper and lower envelope of the signal will be evaluated by applying the cubic spline interpolation function of the data results from the previous step.

c. Obtaining $\mathrm{m}$ after Mediate the upper and lower envelope. It should be subtracted from the input signal.

d. Evaluate the term condition. If this is respected, then the IMF is the difference between $m$ and the input signal, and the evaluation of the next one the difference between the input signal and the IMF resulted. Otherwise, a repetition of the process on the remaining takes place [20].

Figure 2 shows the EMD algorithm block diagram.

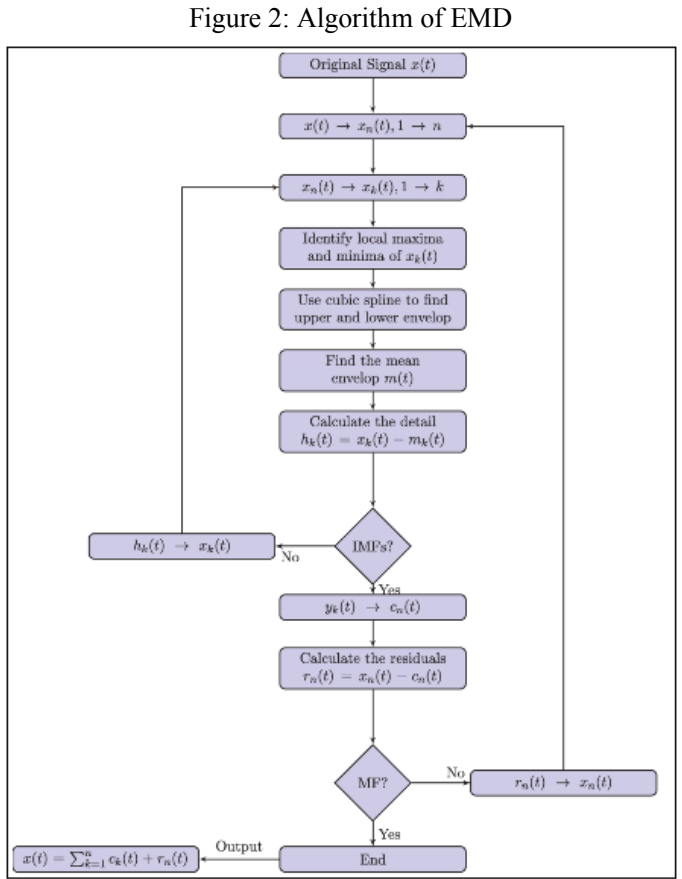

Figure 3: Decomposition Mode of EMD

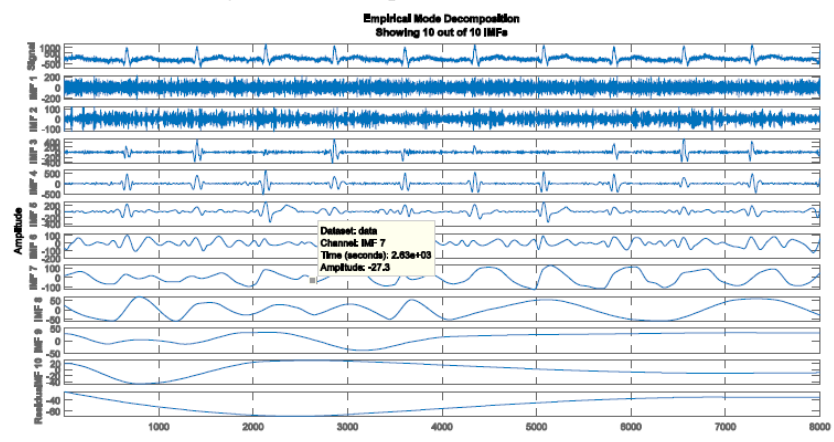

Figure 4: Data Pre-Processing Results
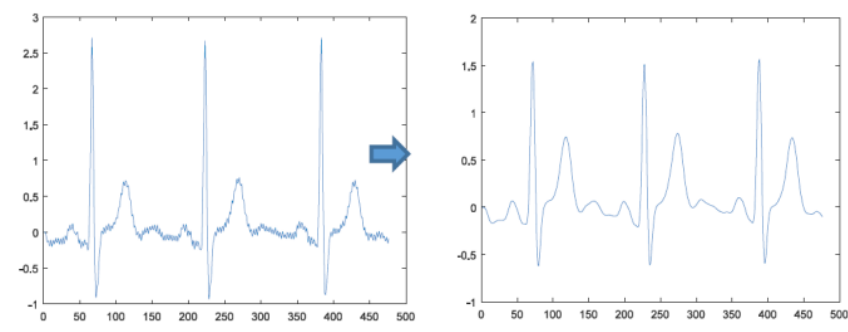

Figure 3 shows us a type of EMD decomposition. Figure 4 shows the results obtained from Pre - Processing step.

\section{Feature Extraction}

The feature extraction stage is the key to the success in using the ECG signal for heartbeat classification. From the cardiac rhythm or directly from the ECG signal's morphology in the time domain and/or in the frequency domain, the feature can be extracted in various forms.

Figure 5 shows the proposed methodology block diagram.

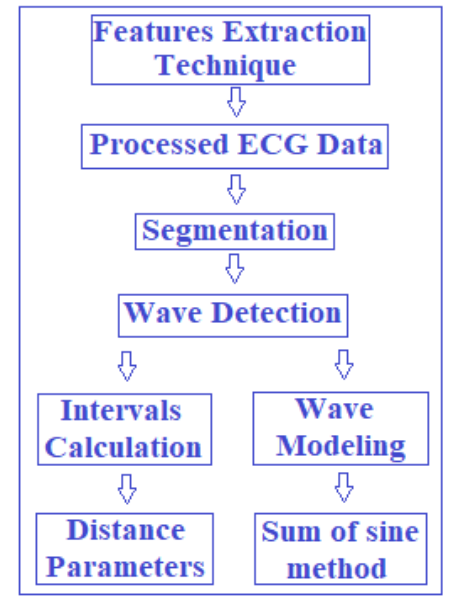

Figure 5: Features Extraction Block Diagram

1) Peak detection: This step is the detection of peaks locations and boundaries of the three waves, the $P$ wave, the QRS complex, and the T wave that all will be used in heartbeat signals ${ }^{[1]}$. Detection of R-peak in ECG is a critical task since this peak is the major characteristic wave. After detecting this peak location, other components of the wave: P, Q, S, and T will be detected by taking R-peak location as a reference and tracing from $R$ peak relative position as shown in figure 6 .

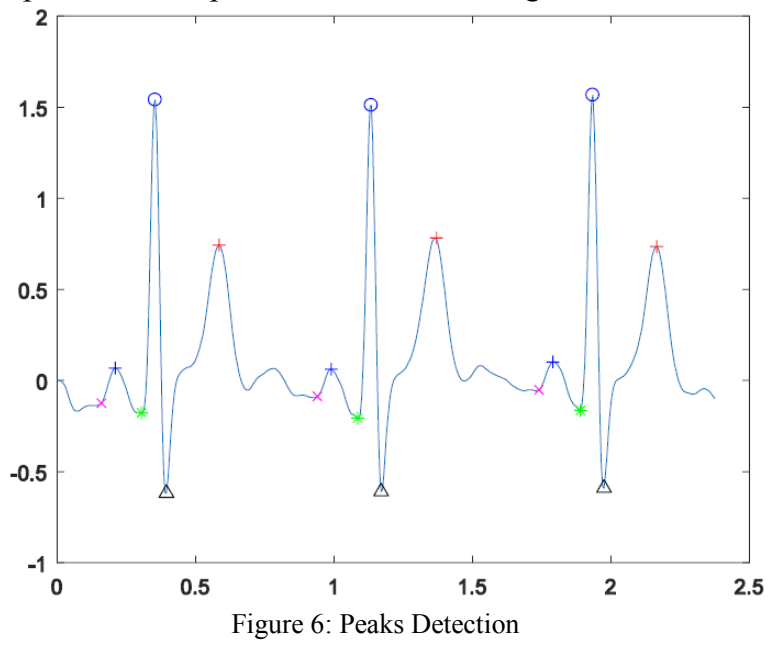


2) Segmentation: After detecting the points, the ECG waveform is segmented into an individual heartbeat, as shown in figure 7 , and decomposed in the sine waveform, as shown in figure 8 .
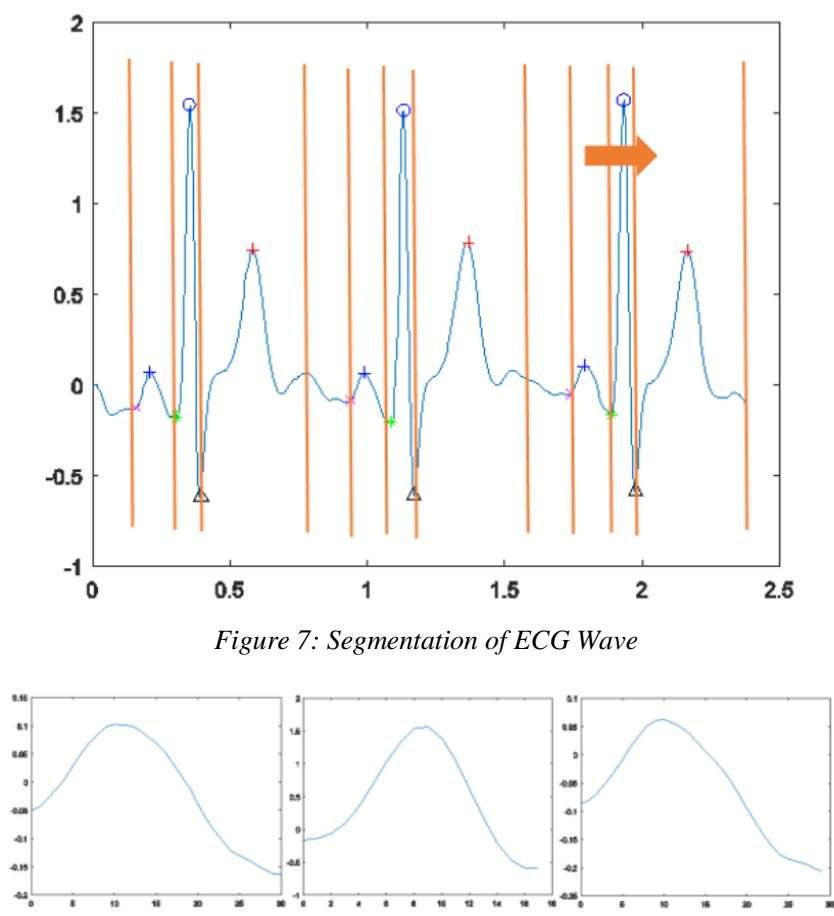

Figure 8: $\mathrm{P}$ wave, QRS wave, and T wave

3) Wave Modeling: The feature extraction aims to have the same template from the same subject and different templates from different subjects. This is a basic point in a biometric heartbeat since in this step the input will be the vector features extracted from heartbeat signals. For curve fitting best model choice we use "cftool" in Matlab and after that, we generate the code. Equation (1) shows the degree 5 of the sum of the sine method that is used for modeling that is shown in Figure 9.

$$
\begin{gathered}
f(x)=a_{1} \sin \left(b_{1} x+c_{1}\right)+a_{2} \sin \left(b_{2} x+c_{2}\right)+ \\
a_{3} \sin \left(b_{3} x+c_{3}\right)+a_{4} \sin \left(b_{4} x+c_{4}\right)+a_{5} \sin \left(b_{5} x+c_{5}\right)
\end{gathered}
$$

Where ai, bi and ciare the coefficients that are used as an input for the artificial neural network.

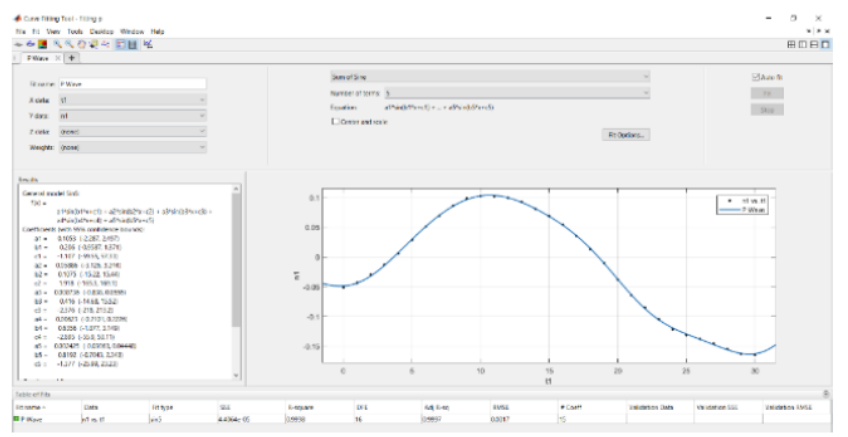

Figure 9: P Wave Modeling

\section{Biometric Template in Database}

In this step, a Database is created for the storage of the biometric features of individuals. Then for decision making, the data that was stored is compared with the input data from the verification phase to find matches ${ }^{[1]}$.

\section{E. Classification}

Classification is used to classify the input data in different sets of classes that would be easy to compare with stored data. In our work, biometric identification is done by input the data of ECG features to two types of Artificial Neural Network (ANN) which are the Multi-layer Perceptron (MLP) and Radial Basis Function (RBF). ECG data of different persons is primarily used for the neural network to be trained. Then for biometric identification of the individuals, the generated neural network from the training will be used.

Figure 10 shows the architecture of Multi-layer Perceptron where this type of ANN has three or more layers that classify data which cannot be linearly separated (i.e., go through a straight path). Thus, it is fully connected, which means that each node within a layer is connected to the succeeding node in the next layer.

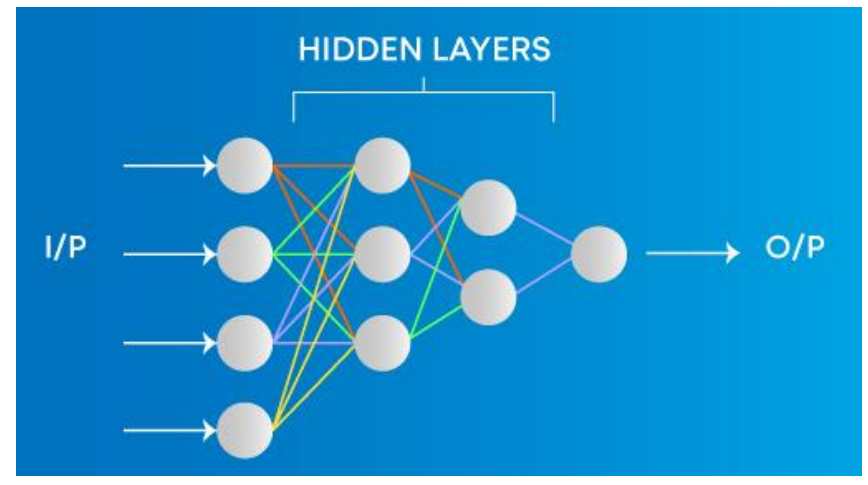

Figure 10: MLP Architecture

a) Advantages of MLP:

- Used for deep learning

\section{b) Disadvantages of MLP:}

- Comparatively complex to design.

- Comparatively slow.

Figure 11 shows the architecture of the Radial Basis Function where this type of ANN only has three layers the input layer, the hidden layer, and the output layer. It is limited to a single hidden layer compared with other ANN types. The hidden layer is hidden in between input and output layers, and it reduces redundancies in data. Compared with other ANN types that can have several hidden layers, learning is faster in an RBFNN. 


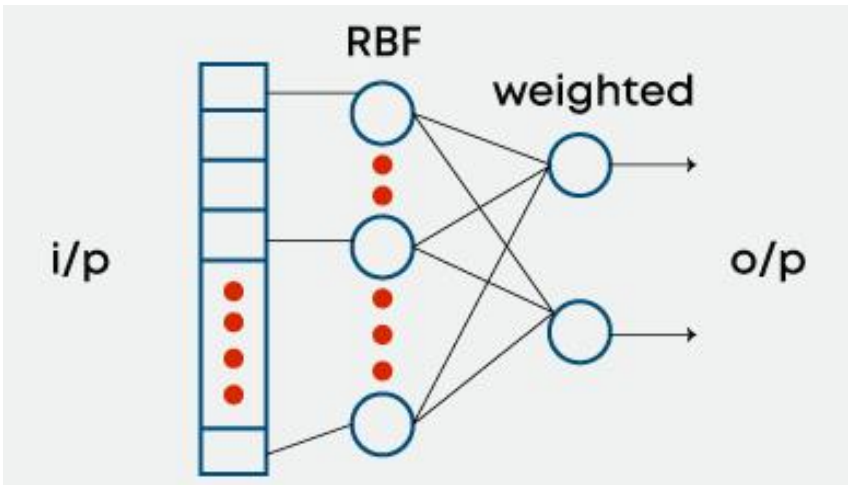

Figure 11: RBF Architecture

To further understand what the different ANN layers are for, imagine that you want to inform your computer that the picture it is shown depicts a car. For the computer to understand, it needs separate tools (or layers). Your car detector can thus have a wheel detector so it can tell something has wheels. It should have a vehicle body detector, which could allow it to differentiate a car from a truck, and a size detector so it can do likewise. These are just some elements that make up hidden layers in artificial neural networks. They do not present the entire image but are parts of it.

\section{F. Decision}

The last step of the heartbeat biometric system is a decision that shows the result of inputted data for verification to YES or NO. After data acquisition, pre-processing, segmentation, feature extraction, and classification steps, a comparison occurred between the information which is collected, with the data that was stored in the database, and this comparison is achieved by the heartbeat biometric system to make the final decision. Then the person will be accepted or denied by the system.

Figure 12 represents its block diagram.

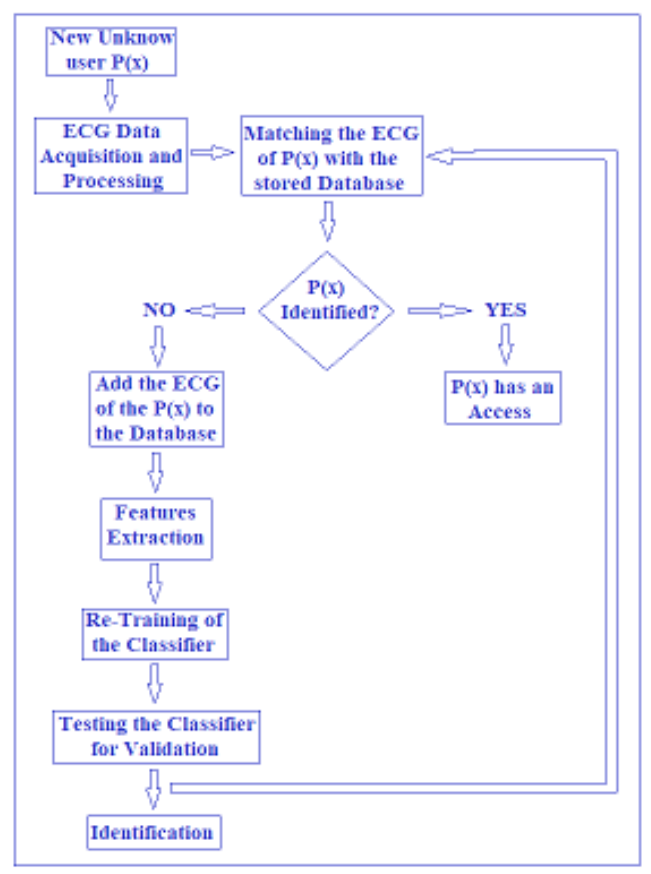

Figure 12: Identification

\section{RESULTS AND DISCUSSION}

The proposed study focuses on an effective algorithm of feature extraction that is based on extracting features depending on the waves modeling of each heartbeat in the ECG signal than by ANN the feature set is examined for Pattern Recognition classifier. Our results, if compared to the ones resulted from extracting features of classical parameters (P, Q, R, S and T peaks) are considered very accurate since the classical parameters' features change per physiological and mental conditions such as exercise, stress, and other diseases like tachycardia which may lead to change in the heart rate [21]. The changes in the heart rate accordingly vary the features such as RR interval, PR interval, and QT interval that contribute to misclassification. Therefore, the most efficient and accurate way to have good results in classification is using wave modeling for feature extraction.
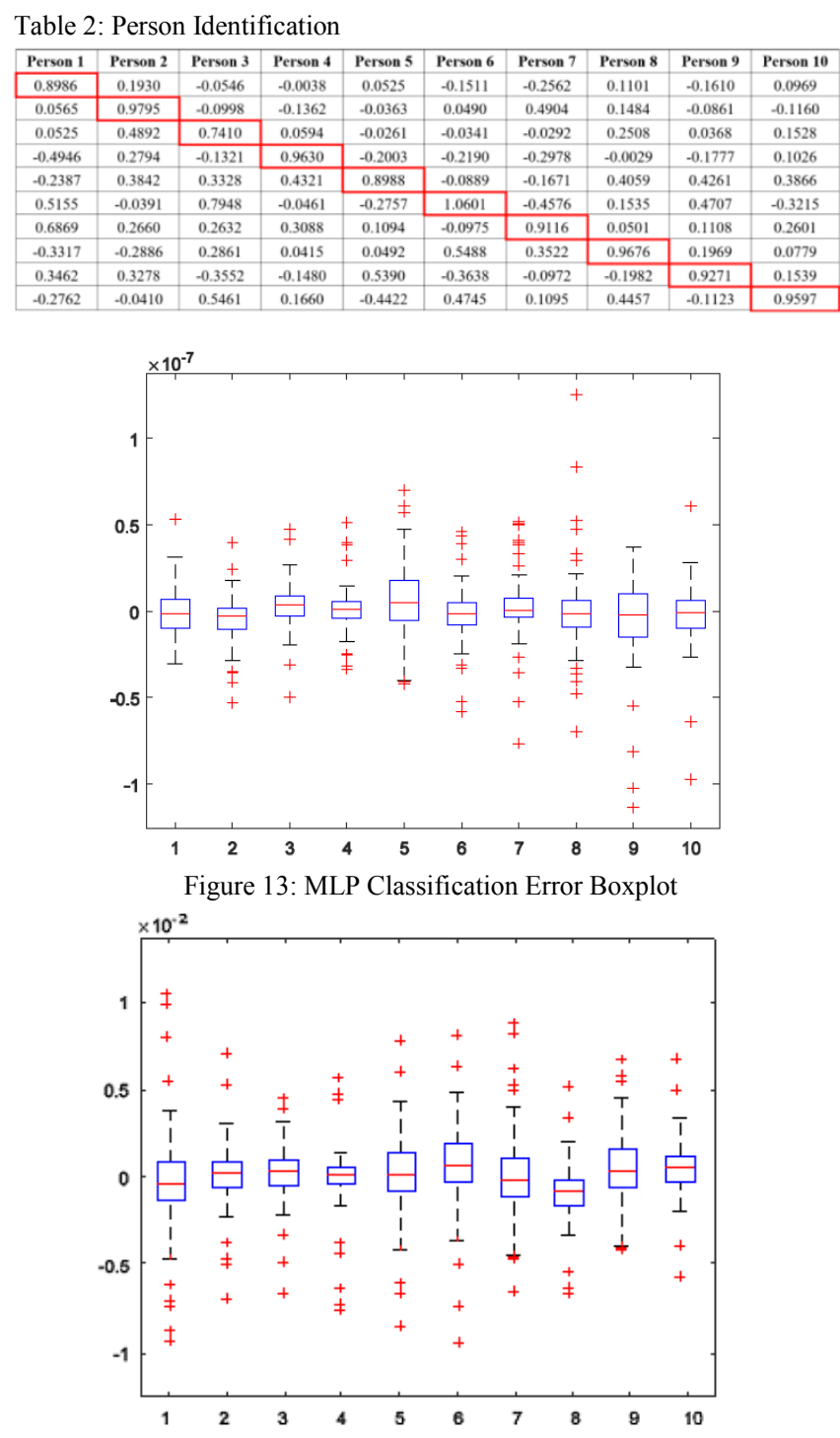

Figure 14: RBF Classification Error Boxplot

Figure 13 and figure 14 illustrate the boxplots, where Figure 13 illustrates the MLP error boxplot which shows the difference between the medians of the degree of variation in distance. It is shown that the error between the real and desired output is negligible (in order of $10^{-7}$ ). This proves that the used methodology can classify persons. The target or 
output is a vector of 10 -elements with a ' 1 ' in the classified position and ' 0 ' everywhere else as shown in table 2 . Besides, the MLP results are better than the RBF results shown in figure 14 .

Although the training is faster in the RBF network classification is slow in comparison to Multi-layer Perceptron.

\section{CONCLUSION}

Since ECG is not imitated, it can precisely identify an individual and can offer a more robust and effective human identification system. This paper gives an overview of the main steps in ECG signal analysis from de-noising ECG, to characteristic points' identification, feature extraction, and effective feature extraction and finally classification to have more accurate identification and classification process of persons. The review identifies different methods of features extraction of the signals from heartbeat and compared based on the accuracy result. A good feature extraction methodology can accurately work for Biometric applications.

\section{ACKNOWLEDGMENT}

This work achieves its success mainly due to the collaboration between the institutions involved (Islamic University of Lebanon and Beirut Arab University). The author would like to acknowledge the continuous support of Dr. Mohammad Ayache, the director of the Master Degrees at the Islamic University of Lebanon, Faculty of Engineering, Thanks also to Dr. Alaa Daher from Beirut Arab University, department of biomedical engineering for his deep follow-up and guidance on this research.

\section{REFERENCES}

[1] Ala Abdulhakim Alariki, Sayed Mahmoud Alavy, Mohammad Reza Yousufi, Mohammad Tareq Aziz and Christine Murray, A Review Study of Heartbeat Biometric Authentication, Volume 13, Number 8, August 2018

[2] Kiran KumarPatro and P. RajeshKumar, Effective Feature Extraction of ECG for Biometric Application, Procedia Computer Science, Volume 115, 2017, Pages 296-306

[3] Biel L, Pettersson O, Philipson L, Wide P. ECG analysis: a new approach in human identification. IEEE Trans Instrum Meas. 2001;50(3):808-12 (IEEE).

[4] Shen TW, Tompkins WJ, Hu YH. One-lead ECG for identity verification. Proceedings of the 2nd Joint, 24th Annual Conference and the Annual Fall Meeting of the Biomedical Engineering Society EMBS/BMES Conference on Engineering in Medicine and Biology; 2002.

[5] Israel SA, Irvine JM, Cheng A, Wiederhold MD, Wiederhold BK. ECG to identify individuals. Pattern Recognition. 2005;

[6] Gahi Y, Lamrani M, Zoglat A, Guennoun M, Kapralos B, ElKhatib K. Biometric identification system based on electrocardiogram data. New Technologies, Mobility and Security
(NTMS); 2008

[7] S. I. Safie, J. J. Soraghan, and L. Petropoulakis, "Electrocardiogram (ECG) biometric authentication using pulse active ratio (PAR)," IEEE, Dec. 2011.

[8] F.-T.-Z. Iqbal, K. A. Sidek, N. A. Noah, and T. S. Gunawan, "A comparative analysis of QRS and cardioid graph based ECG biometric recognition in different physiological conditions," in Proc. IEEE Int. Conf. Smart Instrum., Meas. Appl. (ICSIMA), Nov. 2014.

[9] Sriram JC, Shin M, Choudhury T, Kotz D. Activity-aware ECG-based patient authentication for remote health monitoring. Proceedings of the 2009 International Conference on Multimodal Interfaces; 2009 Nov.

[10] Abdelraheem M, Selim H, Abdelhamid TK. Human identification using the main loop of the vector cardiogram. Am J Signal Process. 2012;

[11] Plataniotis KN, Hatzinakos D, Lee JKM. ECG biometric recognition without fiducial detection. In: Biometrics symposium: special session on research at the biometric consortium conference, 2006. IEEE. 2006.

[12] Chiu CC, Chuang CM, Hsu CY. A novel personal identity verification approach using a discrete wavelet transform of the ECG signal. International Conference on Multimedia and Ubiquitous Engineering (MUE); 2008 Apr.

[13] Chan AD, Hamdy MM, Badre A, Badee V. Wavelet distance measure for person identification using electrocardiograms. IEEE Transactions on Instrumentation and Measurement. 2008;

[14] Zeng F, Tseng KK, Huang HN, Tu SY, Pan JS. A new statistical-based algorithm for ECG identification. 8th International Conference on Intelligent Information Hiding and Multimedia Signal Processing (IIH-MSP); 2012 Jul.

[15] M. N. Dar, M. U. Akram, A. Shaukat, and M. A. Khan, 'ECG based biometric identi_cation for population with normal and cardiac anomalies using hybrid HRV and DWTfeatures," in Proc. 5th Int. Conf. IT Converg. Secur. (ICITCS), Aug. 2015.

[16] J. R. Pinto, J. S. Cardoso, A. Lourenço, and C. Carreiras, "Towards a continuous biometric system based on ECG signals acquired on the steering wheel,", 2017.

[17] H. J. Kim and J. S. Lim, "Study on a biometric authentication model based on ECG using a fuzzy neural network," 2018.

[18] Yu HUANG and Cheng-zhe XU, Research on the Human Identification Algorithm Using ECG Signal, Dept. of Computer Science and Technology, Yanbian University, Yanji, Jilin 133002, China. 2019 International Conference on Power, Energy, Environment and Material Science (PEEMS 2019).

[19] Gaganpreet Kaur, Dr. Dheerendra Singh and Simranjeet Kaur, Electrocardiogram (ECG) as a Biometric Characteristic: A Review, International Journal of Emerging Research in Management \&Technology ISSN: 2278-9359 (Volume-4, Issue-5)

[20] Rupert Faltermeier, Ingo R. Keck, Ana Maria Tomé and Carlos G. Puntonet, EDM - An Introduction, July 2010, DOI: 10.1109/IJCNN.2010.5596829

[21] Shweta H. Jambukia, Vipul K. Dabhi and Harshadkumar B. Prajapati, Classification of ECG signals using Machine Learning Techniques A Survey, 978-1-4673-6911-415,2015

\section{Creative Commons Attribution License 4.0 (Attribution 4.0 International, CC BY 4.0)}

This article is published under the terms of the Creative Commons Attribution License 4.0

https://creativecommons.org/licenses/by/4.0/deed.en_US 